\title{
Childhood neuropsychological deficits associated with adult obsessive-compulsive disorder
}

\author{
Jessica R. Grisham, Tracy M. Anderson, Richie Poulton, Terrie E. Moffitt and Gavin Andrews
}

\section{Background}

Existing neuropsychological studies of obsessive-compulsive disorder (OCD) are cross-sectional and do not provide evidence of whether deficits are trait-related (antecedent and independent of symptomatology) or state-related (a consequence, dependent on symptomatology).

\section{Aims}

To investigate whether there are premorbid neuropsychological deficits associated with adult OCD.

\section{Method}

Longitudinal data were collected from participants of the Dunedin Multidisciplinary Health and Developmental study. Neuropsychological data collected at age 13 were linked with age 32 diagnosis of OCD.

\section{Results}

The group who had OCD at age 32 differed significantly from the control group with no OCD on their performance at age 13 on neuropsychological tests of visuospatial, visuoconstructive and visuomotor skills, controlling for gender and socioeconomic status, but did not differ on tests of general IQ or verbal ability. Performance of the group with OCD on tests of executive functioning was mixed.

\section{Conclusions}

Individuals with OCD have premorbid impairment in visuospatial abilities and some forms of executive functioning, consistent with biological models of OCD.

\section{Declaration of interest}

None.
Cognitive deficits may contribute to the characteristic symptoms of obsessive-compulsive disorder (OCD), such as chronic doubt, repetitive checking, behavioural inflexibility, and ritualistic behaviours. In contemporary neurobiological models of OCD, a specific neural circuit is implicated, comprising the orbitofrontal cortex, the cingulate cortex and the caudate nucleus. ${ }^{1}$ It has been difficult, however, to link findings regarding brain circuit dysfunction and structural differences, cognitive impairment and symptomatology to create a comprehensive, coherent model of OCD. Many studies have attempted to use neuropsychological tests to evaluate deficits in cognitive functioning that may reflect brain functioning impairment associated with OCD. The majority of findings suggest impairment in visuospatial abilities, memory performance and various executive functions, although general intellectual abilities and attention span appear preserved. ${ }^{2}$ This pattern of deficits, however, has not been consistently observed across studies and conclusions regarding specific deficits associated with OCD have not been possible..$^{2-4}$ In addition, almost all of the existing neuropsychological studies of OCD are cross-sectional and do not provide evidence of whether the deficits preceded the onset of the disorder. Discriminating between deficits that play an aetiologically significant role and those that may result from symptomatology has far-reaching theoretical and clinical implications.

The current study used longitudinal data to extend the literature on the relationship between OCD and neuropsychological functioning. We tested the hypothesis that deficit neuropsychological test performance at age 13 would predict OCD diagnosed at age 32. On the basis of biological models and cross-sectional studies, we hypothesised that the strongest associations between childhood neuropsychological test performance and adult OCD would be within the visuospatial/visuomotor and executive domains, whereas general IQ and verbal ability would be unimpaired.

\section{Method}

\section{Participants}

Participants are members of the Dunedin Multidisciplinary Health and Development Study. ${ }^{5}$ Of infants born in Dunedin, New Zealand between April 1972 and March 1973, 1037 children ( $91 \%$ of eligible births; $52 \%$ male) participated in the first follow-up at age 3, constituting the base sample for the longitudinal study. Participants represent the full range of socioeconomic status in the general population of New Zealand's South Island and are primarily White. Participants attend the research unit for a full day of individual data collection. The Otago Ethics Committee approved each phase of the study. Study members gave written informed consent before participating. Assessments were undertaken at ages $3,5,7,9,11,13,15,18,21,26$ and most recently at age 32 when we assessed $96 \%$ of the 1015 study members still alive in 2004-2005. This paper examined participants who were administered neuropsychological tests at age $13(n=740)$, and who received a psychiatric interview for OCD at age 32 years $(n=962)$. Overall, complete data for both measures were available for 700 participants ( $68 \%$ of the original cohort). The children who underwent neuropsychological testing did not differ significantly from the remainder of the cohort on measures of family socioeconomic status, IQ, gender or behaviour problems.

\section{Measures}

Neuropsychological testing at age 13

Two clinical psychologists administered a neuropsychological test battery to study members in two 50-minute sessions, separated by a break and given in counterbalanced order. The battery comprised eight subtests of the Weschsler Intelligence Scales for 
Children (WISC-R) ${ }^{6}$ and seven other neuropsychological tests: Rey-Osterrieth Complex Figure Test (ROCF), Rey Auditory Verbal Learning Test (RAVLT; four trials), Wisconsin Card Sorting Test (WCST; three categories), WISC-R Mazes (an optional WISC-R subtest), Trail Making Test (TMT), Grooved Pegboard Test and the Verbal Fluency Controlled Oral Word Association Test (COWAT). See Lezak et al for a description of each measure. ${ }^{7}$ All of the tests were administered according to standard protocol with three exceptions: the Information subtest was modified for local relevance in the New Zealand version of the WISC-R and the psychologists administered abbreviated versions of the WCST (three categories instead of six) and the RAVLT (four learning trials instead of five). WISC-R factor scores were computed to examine individual psychological dimensions. ${ }^{8}$

\section{Psychiatric status}

At age 11 the study participants were administered the Diagnostic Interview Schedule for Children ${ }^{9}$ to assess psychiatric status. At age 32 OCD symptoms were assessed using a modified version of the Diagnostic Interview Schedule for DSM-IV (DIS-IV). ${ }^{10}$ The DIS-IV has demonstrated good interrater reliability and validity in this cohort. ${ }^{11}$

\section{Statistical analysis}

Test scores were standardised so that the mean was zero and s.d. $=1$. Univariate logistic regression equations were performed to evaluate group differences on neuropsychological test performance. For each of the tests, we compared individuals with OCD at 32 with those who did not have OCD at age 32. All regression coefficients were adjusted for gender and family socioeconomic status. The strength of the association between test performance and diagnostic outcomes was evaluated using odds ratios (ORs) with $95 \%$ confidence intervals (CIs).

\section{Results}

\section{Psychiatric symptoms}

At age 32,13 of the 700 participants (1.9\%) met DSM-IV criteria for OCD. ${ }^{12}$ Individuals in this group reported obsessions related to relatives being hurt or killed (23\%), shameful thoughts $(23 \%)$, harming their loved ones (15\%), accidental harm (15\%) and contamination (15\%). Compulsions reported included checking (62\%), counting/touching (54\%), repetition (23\%) and washing $(15 \%)$.

Most of the OCD group (84.6\%) received one or more additional Axis I diagnoses at age 32. The most common current comorbid diagnoses were depression $(53.8 \%)$, panic disorder $(38.5 \%)$, generalised anxiety disorder $(38.5 \%)$, specific phobia $(30.8 \%)$ and social phobia $(23.1 \%)$. In addition, a portion of the OCD group $(23.1 \%)$ had a current substance dependence diagnosis.

\section{Neuropsychological performance}

Independent samples $t$-tests were conducted to examine group differences in overall intellectual functioning at age 13. The small difference (as measured by prorated WISC-R full-scale IQ) between those with (mean 103.31, s.d. $=18.04$ ) and without (mean 108.09 , s.d. $=14.36)$ OCD at age 32 was not significant $(t(685)=1.18, P=0.24)$.

Table 1 contains neuropsychological test results for cohort members with and without a diagnosis of OCD at age 32. Odds ratios show the effect of neuropsychological performance at age 13 on risk for OCD at age 32, controlling for socioeconomic status and gender. Although there were no group differences on the WISC-R freedom from distractibility or verbal comprehension factors, performance of the OCD group on the WISC-R perceptual organisation factor was 0.75 s.d. lower than that of

\begin{tabular}{|c|c|c|c|}
\hline Variable & $\begin{array}{c}\text { No OCD, mean (s.d.) } \\
\qquad(n=687)\end{array}$ & $\begin{array}{l}\text { OCD, mean (s.d.) } \\
\quad(n=13)\end{array}$ & $\begin{array}{l}\text { No OCD v. OCD, } \\
\text { OR }(95 \% \mathrm{Cl})^{\mathrm{b}}\end{array}$ \\
\hline \multicolumn{4}{|l|}{ WISC-R factors, $z$-score } \\
\hline Perceptual organisation & $0.02(0.96)$ & $-0.73(1.12)$ & $0.4(0.25-0.76)^{* *}$ \\
\hline Freedom from distractibility & $-0.03(0.98)$ & $0.07(1.03)$ & $1.1(0.62-2.03)$ \\
\hline Verbal comprehension & $-0.01(0.96)$ & $-0.15(1.31)$ & $0.8(0.45-1.49)$ \\
\hline \multicolumn{4}{|l|}{ Rey-Osterrieth Complex Figure Test, z-score } \\
\hline Copy, & $0.05(0.90)$ & $-0.77(1.26)$ & $0.6(0.40-0.82)^{* *}$ \\
\hline Delayed recall & $0.03(1.00)$ & $-0.88(0.68)$ & $0.4(0.24-0.71)^{* *}$ \\
\hline \multicolumn{4}{|l|}{ Rey Auditory -Verbal Learning Test, z-score } \\
\hline Trials 1-4 & $0.00(1.00)$ & $-0.22(1.18)$ & $0.8(0.47-1.37)$ \\
\hline Post interference test & $0.01(1.00)$ & $-0.44(1.20)$ & $0.7(0.39-1.09)$ \\
\hline Delayed recall & $0.02(0.99)$ & $-0.32(1.23)$ & $0.7(0.41-1.22)$ \\
\hline \multicolumn{4}{|l|}{ Wisconsin Card Sorting Test, z-score } \\
\hline Total errors & $-0.01(1.00)$ & $0.49(1.02)$ & $1.6(0.94-2.63)$ \\
\hline Perseverative errors & $0.00(1.00)$ & $0.05(1.21)$ & $1.1(0.62-1.80)$ \\
\hline \multicolumn{4}{|l|}{ Trail Making Test (TMT) A and B, Z-score } \\
\hline TMT B, S & $0.00(1.02)$ & $0.15(0.86)$ & $1.1(0.77-1.58)$ \\
\hline TMT B-A, S & $0.00(1.02)$ & $0.15(1.01)$ & $1.1(0.61-1.11)$ \\
\hline \multicolumn{4}{|l|}{ Grooved Pegboard Test, z-score } \\
\hline Right hand & $-0.02(1.00)$ & $0.93(2.00)$ & $1.5(1.11-1.94)^{* *}$ \\
\hline Left hand & $-0.02(0.95)$ & $0.59(1.15)$ & $1.6(1.08-2.45)^{*}$ \\
\hline WISC-R Mazes, Z-score & $0.04(0.95)$ & $-0.51(1.14)$ & $0.6(0.38-0.97)^{*}$ \\
\hline Verbal Fluency Controlled Oral Word Association Test, z-score & $0.00(1.00)$ & $-0.20(1.00)$ & $0.8(0.45-1.44)$ \\
\hline $\begin{array}{l}\text { WISC-R, Wechsler Intelligence Scale for Children-Revised } \\
\text { a. } n \text { varies slightly for some risk factors owing to missing data. } \\
\text { b. Adjusted for gender and socioeconomic status. } \\
{ }^{*} P<0.05 .{ }^{* \star} P<0.01 \text {. }\end{array}$ & & & \\
\hline
\end{tabular}


the control group. Performance on the ROCF was also significantly worse in the OCD group (0.82 s.d. and 0.91 s.d. lower for the copy and delayed recall conditions, respectively), as was performance on WISC-R Mazes (0.55 s.d. lower for the OCD group than for the control group). Finally, the OCD group was significantly slower than the control group on the Grooved Pegboard test. The Pegboard effect persisted following adjustment for hand preference. No significant differences were found between groups on the COWAT, the TMT, the RAVLT or the WCST. However, on WCST total errors the OCD group scored 0.5 s.d. below controls. This difference represents a moderate effect size, but was not significant because of the small size of the OCD group.

\section{Discussion}

We found a relationship between premorbid impairment in specific cognitive domains at age 13 and OCD at age 32 . Compared with the rest of the cohort, the OCD group demonstrated poor performance on tests that assess visuospatial and visuomotor ability but performance on tests of verbal comprehension, verbal fluency and verbal memory was comparable with that of controls. Performance of the OCD group on tests of executive functioning, such as planning, cognitive flexibility and set-shifting, was mixed. Given evidence of gender dimorphism in OCD, ${ }^{13}$ it is significant that these effects persisted despite controlling for gender.

Although there are no comparable prospective studies, on the basis of cross-sectional studies of neuropsychological functioning and OCD, we expected impairment on tasks that reflect visuospatial, visuoconstructive and visuomotor skills and non-verbal memory. ${ }^{4}$ The poor performance observed across the ROCF, Grooved Pegboard, WISC-R Mazes and the WISC perceptual organisation factor supported our predictions. We also expected to observe some relation between executive skills and OCD; however, the findings were divergent. Performance of the OCD group on tests that evaluated both visuospatial/visuomotor skills and executive functioning (ROCF, WISC-R Mazes) was impaired, whereas performance on tests that evaluated other executive functions such as set-shifting and divided attention (WCST, TMT) was comparable between the groups. The equivalent performance of the OCD group on the WISC-R full-scale IQ, as well as on the WISC-R verbal tests, the TMT and the WCST, suggests that there is a pattern of specific deficits associated with OCD, rather than overall cognitive or neuropsychological impairment.

A previous study of OCD with this same birth cohort at age 18 found that the OCD group did not differ significantly on any of the neuropsychological tests at age $13 .{ }^{14}$ Changes in the OCD criteria from DSM-III ${ }^{15}$ to DSM-IV have reduced the reported prevalence of the disorder in the general population. ${ }^{16}$ The application of the slightly more stringent DSM-IV diagnostic criteria may have resulted in the identification of a more valid OCD group; only $1.9 \%$ of age 32 study members met criteria for OCD compared with the $4 \%$ prevalence rate in the earlier study. ${ }^{14}$ In addition, from a developmental perspective, study members who were diagnosed with OCD at age 32 may represent a more severe and persistent subgroup.

A limitation to the current study was the small number of study members who had OCD outcomes at age 32 . In addition, although the findings suggest impairment in visuospatial and visuomotor performance and non-verbal memory, neuropsychological tests assess performance in more than one domain. For example, memory dysfunction on the ROCF may be secondary to impaired executive functioning, in particular the inability to apply efficiently elaborated strategies. ${ }^{17}$ In addition, consistent with high rates of comorbidity generally found among mood and anxiety disorders, ${ }^{18}$ the majority of the OCD group met criteria for an additional Axis I disorder. This introduces the possibility that differences between the OCD and the comparison group were partially accounted for by the presence of comorbid psychopathology. Finally, although neuropsychological measures provide clues about neuroanatomical substrates, we cannot directly conclude neurobiological dysfunction from poor test performance.

Despite these limitations, the current findings are noteworthy because of the unique longitudinal nature of the study. We have identified associations between childhood neuropsychological test performance and adult OCD that span two decades. We found selective deficits in visuospatial and visuoconstructive abilities and non-verbal memory, with intact verbal functioning and ambiguous frontal lobe functioning. Theories arguing that developmental disruption to brain maturation processes (such as abnormal postnatal pruning in the ventromedial prefrontal cortex) contributes to $\mathrm{OCD}^{19}$ may view these premorbid childhood deficits as support in that individuals were exhibiting neuropsychological deficits before they had developed the full OCD syndrome. It is important to note that OCD is likely to be multiply determined ${ }^{20}$ and not all participants with these deficits developed OCD. Consistent with a diathesis-stress model of OCD, whether particular neuropsychological deficits result in the onset of OCD may depend on psychosocial factors, such as parenting styles or critical stressful life events.

Jessica R. Grisham, PhD, School of Psychology, University of New South Wales, Sydney, Australia; Tracy M. Anderson, PhD, School of Psychiatry, University of New South Wales, Sydney, Australia; Richie Poulton, PhD, Dunedin School of Medicine, University of Otago, New Zealand; Terrie E. Moffitt, PhD, Institute of Psychiatry, King's College London, UK and Duke University, Durham, NC, USA; Gavin Andrews, MD, School of Psychiatry, University of New South Wales, Sydney, Australia

Correspondence: Jessica Grisham, School of Psychology, University of New South Wales, Sydney, Australia 2052. Email: jgrisham@psy.unsw.edu.au

First received 1 Jul 2008, final revision 15 Sep 2008, accepted 15 Jan 2009

\section{Funding}

The Dunedin Multidisciplinary Health and Development Research Unit is supported by the New Zealand Health Research Council. This research was supported by grants from the US National Institute of Mental Health (MH 45070, MH49414), the UK Medical Research Council grant G0100527, and a Postdoctoral Fellowship with the School of Psychiatry, University of New South Wales.

\section{Acknowledgements}

We thank study founder Dr Phil Silva and the study members, and their families and friends for continued support.

\section{References}

1 Insel TR. Toward a neuroanatomy of obsessive-compulsive disorder. Arch Gen Psychiatry 1992; 49: 739-44.

2 Greisberg S, McKay D. Neuropsychology of obsessive-compulsive disorder: a review and treatment implications. Clin Psychol Rev 2003; 23: 95-117.

3 Simpson HB, Rosen W, Huppert JD, Lin S, Foa EB, Liebowitz MR. Are there reliable neuropsychological deficits in obsessive-compulsive disorder? J Psychiatr Res 2006; 40: 247-57.

4 Kuelz AK, Hohagen F, Voderholzer U. Neuropsychological performance in obsessive-compulsive disorder: a critical review. Biol Psych 2004; 65 185-236.

5 Frost LA, Moffitt TE, McGee R. Neuropsychological correlates of psychopathology in an unselected cohort of young adolescents. J Abnorm Psychol 1989; 98: 307-13 
6 Wechsler D. Manual for the Wechsler Intelligence Scale for Children Revised. Psychological Corporation, 1974.

7 Lezak MD, Howieson DB, Loring DW. Neuropsychological Assessment (4th edn). Oxford University Press, 2004

8 Kaufman A. Factor analysis of the WISC-R at age levels between 6.5 and 16.5 years. J Consult Clin Psychol 1975; 43: 135-47.

9 Costello A, Edelbrock C, Kalas R, Kessler M, Klaric SA. The National Institute of Mental Health Diagnostic Interview for Children (DISC). National Institute of Mental Health, 1982

10 Robins LN, Cottler L, Bucholz K, Compton W. Diagnostic Interview Schedule for DSM-IV. Washington University Press, 1995.

11 Newman DL, Moffitt TE, Caspi A, Magdol L, Silva PA, Stanton WR. Psychiatric disorder in a birth cohort of young adults: prevalence, comorbidity, clinical significance, and new case incidence from ages 11 to 21. J Consult Clin Psychol 1996; 64: 552-62.

12 American Psychiatric Association. Diagnostic and Statistical Manual of Mental Disorder (4th edn) (DSM-IV). APA, 1994.

13 Zohar $\mathrm{AH}$, Gross-Isseroff $\mathrm{R}$, Hermesh $\mathrm{H}$, weizman $\mathrm{A}$. Is there sexual dimorphism in obsessive-compulsive disorder? Neurosci Biobehav Rev 1999: 23: 845-9.
14 Douglass HM, Moffitt TE, Dar R, McGee R, Silva P. Obsessive-compulsive disorder in a birth cohort of 18-year-olds. Prevalence and predictors. J Am Acad Child Adolesc Psychiatry 1995; 34: 1424-31.

15 American Psychiatric Association. Diagnostic and Statistical Manual of Mental Disorders (3rd edn) (DSM-III). APA, 1980.

16 Crino R, Slade T, Andrews G. The changing prevalence and severity of obsessive-compulsive disorder criteria from DSM-III to DSM-IV. Am J Psychiatry 2005; 162: 876-82.

17 Savage CR, Keuthen NJ, Jenike MA, Brown HD, Rauch SL, Jenike MA. Organizational strategies mediate nonverbal memory impairment in obsessive-compulsive disorder J Neuropsychiatry Clin Neurosci 1999; 8: 99-103.

18 Brown TA, Campbell LA, Lehman CL, Grisham JR, Mancill RB. Current and lifetime comorbidity of the DSM-IV anxiety and mood disorders in a large clinical sample. J Abnorm Psychol 2001; 110: 585-99.

19 Rosenberg DR, Keshavan MS. Toward a neurodevelopmental model of obsessive-compulsive disorder. Biol Psych 1998; 43: 623-40.

20 Grisham JR, Anderson TM, Sachdev PS. Genetic and environmental influences on obsessive-compulsive disorder. Eur Arch Psychiatry Clin Neurosci 2008; 258: $107-16$

\section{0 words}

\section{My prison experience}

\section{Bernice Knight}

Visiting an inner-city prison as a medical student, I was unsure how I would respond to this mass incarceration of life. The anxiety manifesting in my stomach as I passed through the entrance gate, the fear of how inmates would respond to me and of names they might call. Corridors were cold, stark, echoic, with a constant reminder of inmates' plight to end their lives in the endless safety netting; calls from unknown locations and cells with no relief. I don't have a mental illness, yet I felt anxious and paranoid. It left me very concerned for those that do. 